\title{
AVALIAÇÃO DA SOBRECARGA EM FAMILIARES CUIDADORES DE PACIENTES ESQUIZOFRÊNICOS ADULTOS
}

\author{
Manoel Dias de Souza Filho* \\ Andréia de Oliveira Sousa ${ }^{\#}$ \\ Alexandre Castelo Branco Vaz Parente \\ Maria do Carmo de Carvalho e Martins ${ }^{\circledast}$
}

\begin{abstract}
RESUMO. O estudo avaliou o sentimento de sobrecarga objetiva e subjetiva deflagrado em cuidadores de adultos portadores de esquizofrenia. A coleta de dados foi realizada em um hospital-dia da rede pública da cidade de Teresina-PI - Brasil, e a amostra foi constituída por 14 familiares que cuidavam direta ou indiretamente de portador de esquizofrenia. Verificaram-se sobrecargas objetiva e subjetiva semelhantes nas dimensões relacionadas à assistência na vida cotidiana, à supervisão dos comportamentos problemáticos, ao impacto nas rotinas diárias e a preocupações com o paciente. Observou-se que os cuidadores quase sempre necessitam cobrir despesas desencadeadas pelo doente. Desse modo, destaca-se a necessidade, por parte dos dispositivos em saúde mental, de estratégias que viabilizem o apoio, a orientação e a preparação das famílias no tocante ao gerenciamento do cuidado ao portador de esquizofrenia.
\end{abstract}

Palavras-chave: Esquizofrenia; família; sobrecarga.

\section{SCHIZOPHRENIA AND FAMILY BURDEN}

\begin{abstract}
This study assessed the feeling of being burdened caused when caring for adult schizophrenia patients, with the aim of learning about the objective and subjective burden, as well as describing the socio-economic condition of the participants. The data collection took place at a public Hospital, in Teresina city, Piauí state - Brazil. The sample consisted of 14 family members who care directly or indirectly for a schizophrenic patient. After analyzing the data, it was found that there is a similar objective and subjective overload in areas related to normal day-to-day care, supervision of behavioral problems, impact on daily routines and concerns with the patient. The caregivers almost always need to cover costs that have arisen in connection with the patient. Therefore, it is clear that mental health strategies are needed to enable support, guidance and preparation of families in the management of psychiatric patient care.
\end{abstract}

Key words: Schizophrenia; family; burden.

\section{LA ESQUIZOFRENIA Y SOBRECARGA DE LA FAMILIA}

RESUMEN. El estudio se propone evaluar el sentimiento de sobrecarga objetivo y subjetivo desencadenado al prestar asistencia a los pacientes adultos esquizofrénicos y caracterizar la condición socio económica de los participantes. Los datos fueron recogidos en el Hospital Día en Teresina - Pi - Brasil y la muestra fue constituida por 14 familiares que cuidan directa o indirectamente de pacientes adultos esquizofrénicos. Se verificó la sobrecarga objetiva y subjetiva semejante en las dimensiones relacionadas a la asistencia en la vida cotidiana, supervisión de los comportamientos problemáticos, impacto en las rutinas diarias y preocupaciones con el paciente. También se observó que las personas que cuidan casi siempre tienen que cubrir los gastos provocados por el enfermo, es así que existe la necesidad por parte de las instituciones responsables en salud mental proporcionen estrategias que viabilicen el apoyo, orientación y la preparación de las familias en el gerenciamiento al cuidado del paciente psiquiátrico.

Palabras-clave: Esquizofrenia; familia; sobrecarga.

Professor de Psicofisiologia do Curso de Psicologia e de Ciências Fisiológicas do Curso de Biomedicina da Universidade Federal do Piauí-UFPI-Parnaíba.

\# Psicóloga comunitária do Centro de Referência da Assistência Social, Brasil.

Il Doutor em Medicina da Saúde Mental pela Universidade de São Paulo, Brasil. Professor adjunto da Universidade Federal do Piauí, Brasil.

æ Professora do Departamento de Biofísica e Fisiologia e Membro do Programa de Pós-Graduação em Alimentos e Nutrição da Universidade Federal do Piauí, Professora titular da Faculdade NOVAFAPI. 
Os efeitos sociais da esquizofrenia podem ser devastadores, mas a natureza do seu processo permanece obscura. O diagnóstico deve ser feito considerando em parte a presença de certos sintomas psicológicos, mas também a exclusão de outros (Crow, 1980). O clima afetivo familiar crítico, hostil e de alto envolvimento emocional pode afetar negativamente o curso dessa síndrome, pois, segundo Bentall (2006), a relação entre trauma na infância, perda, estresse, psicose, disfunção social e familiar, abuso e trauma é um importante precursor de transtornos psicóticos.

As intervenções psicossociais em familiares de indivíduos com esquizofrenia desenvolveram-se a partir de estudos nos quais foi demonstrado que a presença de um membro com esquizofrenia na família está relacionada a sobrecarga em diversos aspectos da vida da família, como os relacionamentos, o lazer, a saúde física e mental; e também que quanto maior o sentimento de sobrecarga relatado pelos familiares, maiores as chances de estes serem críticos, hostis e muito envolvidos emocionalmente com o membro doente (Scazufca, 2000).

Com o movimento da reforma psiquiátrica, a família passou a ser a principal provedora dos cuidados necessários no tratamento de pacientes psiquiátricos. Em contrapartida, tais famílias encontram-se esquecidas e sem conhecimento em relação aos males que atingem seu parente, sendo a assistência prestada pelos modelos substitutivos de atenção em saúde mental considerada insatisfatória no seu modo de auxiliá-las no enfrentamento da doença mental (Bandeira, Calzavara \& Varella, 2005).

O termo "sobrecarga familiar" (do inglês "family burden") fundamenta-se no impacto provocado pela presença do paciente em sofrimento mental sobre o ambiente familiar e envolve os aspectos econômicos, práticos e emocionais a que se encontram submetidos os familiares que se encarregam do cuidado necessário e exigido pelo doente (Bandeira \& Barroso, 2005)".

A família pode se sentir culpada pelas dificuldades enfrentadas por seu ente próximo e também apresentar ansiedade por não saber, em suas ações, como lidar com alguns comportamentos apresentados (por exemplo, o silêncio excessivo, a fala contínua e desordenada) ou com a imprevisibilidade. Surge, ainda, insegurança quanto ao melhor modo possível de cuidar do paciente, a esclarecimentos a serem dados às crianças sobre a doença mental e ao contato entre estas e o paciente (Pegoraro \& Caldana, 2006).
Tendo em vista tais fatores, este trabalho teve como objetivo avaliar a sobrecarga (objetiva e subjetiva) experienciada pelo familiar cuidador de adulto portador de esquizofrenia em tratamento no Hospital-Dia Dr. Wilson Freitas em Teresina-PI Brasil para que se possa repensar o atual modelo de assistência prestada pelos serviços de saúde mental, sobre o qual pretendemos gerar alguma contribuição.

\section{METODOLOGIA}

O presente trabalho correspondeu a um estudo observacional transversal que avaliou a sobrecarga de familiares cuidadores de adultos portadores de esquizofrenia atendidos no Hospital-Dia Dr. Wilson Freitas, unidade de saúde mental da Rede Pública do Estado do Piauí integrada ao Sistema Único de Saúde (SUS), em Teresina-PI - Brasil. Os participantes da pesquisa constituíram-se de cuidadores de 14 portadores de esquizofrenia, com idade acima dos 18 anos, de ambos os gêneros, que acompanhavam direta ou indiretamente o tratamento do paciente $\mathrm{e}$ concordaram com os termos pré-determinados na pesquisa. Apesar da reduzida quantidade de participantes do estudo, o número amostral obtido representou todos os cuidadores de pacientes esquizofrênicos atendidos nesse hospital no período de setembro a outubro de 2008. Vale ressaltar que esse número amostral foi determinado pela própria demanda do hospital em relação aos cuidados com os pacientes esquizofrênicos e pela dificuldade de acesso a outros cuidadores de portadores de esquizofrenia, em razão de atualmente existir apenas um hospital psiquiátrico na cidade de Teresina-PI.

A pesquisa seguiu as determinações da resolução 196/96 (Brasil, 1996) e foi aprovada pelo Comitê de Ética em Pesquisa com Humanos da Faculdade Integral Diferencial-FACID (Parecer: 425/08). Cada participante foi devidamente informado a respeito dos objetivos da pesquisa e da garantia de confidencialidade, privacidade e anonimato e de que a não participação ou desistência a qualquer momento da pesquisa não acarretaria prejuízos e/ou constrangimentos, e então confirmou a participação no estudo por meio da assinatura do Termo de Consentimento Livre e Esclarecido.

A coleta de dados foi realizada por meio da Escala de Avaliação da Sobrecarga dos familiares (FBIS-BR), desenvolvida por Schene, Tessler e Ganache (1994), adaptada e validada para o Brasil por Bandeira (Bandeira et al., 2005). A escala FBIS-BR avalia o grau de sobrecarga objetiva e subjetiva dos familiares em cinco dimensões da vida dos familiares, 
que consistem nas seguintes subescalas: A) Assistência na vida cotidiana do paciente, B) Supervisão aos comportamentos problemáticos do paciente, C) Gastos financeiros do familiar com o paciente, D) Impacto nas rotinas diárias da família; e E) Preocupações do familiar com o paciente. A FBISBR avalia ainda o tipo e valor das despesas com os pacientes, a contribuição dos pacientes para cobrir tais despesas e as alterações permanentes ocorridas na vida do cuidador.

Este instrumento se compõe de 52 itens, sendo a sobrecarga objetiva avaliada em escalas Likert ( $1=$ nenhuma vez , até $5=$ todos os dias), indicando a frequência com que o familiar cuidador executou tarefas para o paciente, lidou com seus comportamentos problemáticos e sofreu alterações diárias na vida. A sobrecarga subjetiva é avaliada em escalas Likert de quatro pontos $(1=$ nem um pouco, até $4=$ muito) para o grau de incômodo ao prestar assistência cotidiana e de alterações na vida, e por escala de cinco pontos ( $1=$ nunca, até $5=$ sempre ou quase sempre) para a frequência de preocupações e peso das despesas com os pacientes.

A aplicação da escala FBIS-BR e do questionário sociodemográfico foi realizada mediante a leitura das perguntas para o familiar cuidador, o qual respondeu de acordo com a experiência vivenciada no cuidar cotidiano do adulto portador de esquizofrenia. A aplicação foi feita em entrevista individual com o familiar considerado como principal cuidador realizada no pátio do hospital-dia, pois os familiares preferiram observar de longe seus respectivos enfermos, uma vez que alguns temiam que acontecesse algo com seu paciente ou que este fugisse ou tivesse algum comportamento perturbador se eles se ausentassem do pátio. Como a FBIS-BR não possui ponto de corte estabelecido, a indicação de sobrecarga elevada é feita considerando-se a porcentagem de respostas aos dois últimos pontos das escalas Likert para cada subescala.

A análise dos dados foi feita utilizando-se os softwares EpiInfo versão 6.04d (Dean, Dean, Burton \& Dicher, 1994) e BioStat versão 5.0 (Ayres, Ayres Jr, Ayres \& Santos, 2007). Para verificação da hipótese de nulidade foi utilizado o teste ANOVA (Friedman) e fixado o valor de alfa menor que 5\% como parâmetro de rejeição da hipótese de nulidade.

\section{RESULTADOS}

As características socioeconômicas dos familiares encontram-se na tabela 1 . Verificou-se que em sua maior parte os cuidadores de adultos portadores de esquizofrenia atendidos no citado hospital-dia eram do sexo feminino $(71,42 \%)$, tinham relação conjugal estável $(85,71 \%)$, que metade deles cursara o ensino fundamental completo e nenhum deles concluíra o ensino superior. Além disso, apenas metade dos entrevistados pertencia ao estado do Piauí, enquanto 42,82\% eram oriundos do Maranhão e 7,14\% do Pará (Tabela 1).

Tabela 1. Características Socioeconômicas de Familiares Cuidadores de Portadores de Esquizofrenia Atendidos em um Hospital Psiquiátrico de Teresina-PI

\begin{tabular}{lcc}
\hline Variáveis & \multicolumn{2}{c}{ Total } \\
\cline { 2 - 3 } & $\mathbf{n}$ & $\%$ \\
\hline Sexo & & \\
Feminino & 10 & 71,42 \\
Masculino & 4 & 28,58 \\
Estado civil & & \\
Cônjuge Presente & 12 & 85,71 \\
Solteiro & 2 & 14,29 \\
Escolaridade & & \\
Fundamental Completo & 7 & 50,00 \\
Fundamental Incompleto & 4 & 28,58 \\
Médio Incompleto & 1 & 7,14 \\
Médio Completo & 1 & 7,14 \\
Superior Incompleto & 1 & 7,14 \\
Estado da Federação & & \\
Piauí & 7 & 50,00 \\
Maranhão & 6 & 42,86 \\
Pará & 1 & 7,14 \\
Gastos & & \\
Transporte & 14 & 100,00 \\
Roupas & 8 & 57,14 \\
Pequenos gastos (Trocados) & 8 & 57,14 \\
Alimentação & 13 & 92,85 \\
Moradia & 3 & 21,43 \\
Medicamento & 7 & 50,00 \\
Tratamento de saúde & 5 & 35,72 \\
Outros gastos médicos & 1 & 7,14 \\
Cigarros & 1 & 7,14 \\
Objetos pessoais & 11 & 78,57 \\
Telefone & 11 & 78,57 \\
Pagamento de profissionais para cuidar do paciente & 0 & 0 \\
Plano de saúde & 2 & 14,28 \\
\hline & & \\
\hline
\end{tabular}

No que diz respeito aos gastos financeiros dos entrevistados com os pacientes esquizofrênicos, verificou-se que os cuidadores gastavam com maior frequência em itens como transporte, alimentação, objetos pessoais e telefone, não tendo sido identificados gastos com pagamentos de profissionais para cuidar do paciente (Tabela 2). 
Tabela 2. Média e Desvio Padrão das Variáveis da Idade, do Número de Pessoas do Domicílio dos Familiares Cuidadores, e Tempo da Síndrome de Portadores de Esquizofrenia Atendidos em um Hospital Psiquiátrico de Teresina-PI

\begin{tabular}{|c|c|c|c|c|}
\hline Variáveis & Média & Desvio Padrão & o Mínimo & o Máximo \\
\hline Idade (anos) & 48,35 & 11,47 & 31 & 69 \\
\hline Número de familiares & 5,2 & 1,77 & 2 & 7 \\
\hline Tempo da síndrome (anos) & 11,54 & 8,22 & 0,08 & 26 \\
\hline
\end{tabular}

A Tabela 3 apresenta a sobrecarga objetiva dos familiares relacionada a tarefas de assistência à vida cotidiana executadas mais de três vezes por semana. Observou-se que os cuidadores ajudavam na higiene pessoal dos pacientes $(64,28 \%)$, eram responsáveis pela administração dos medicamentos $(78,57 \%)$ e, nessa mesma proporção, também eram encarregados das tarefas de casa, das compras $(42,85 \%)$, do preparo de alimentos $(85,71 \%)$, do transporte $(78,57 \%)$. Além disso, os cuidadores administravam o dinheiro do paciente $(50,00 \%)$, dedicavam seu tempo livre aos pacientes $(64,28 \%)$ e acompanhavam os doentes nas consultas médicas periódicas $(78,57 \%)$.

Tabela 3. Sobrecarga Objetiva dos Familiares Cuidadores de Portadores de Esquizofrenia Atendidos em um Hospital Psiquiátrico de Teresina-PI

\begin{tabular}{|c|c|c|c|c|c|c|}
\hline & \multicolumn{2}{|c|}{$\begin{array}{c}\text { Respostas } \\
1 \text { e } 2^{\mathrm{a}}\end{array}$} & \multicolumn{2}{|c|}{$\begin{array}{c}\text { Respostas } \\
4 \text { e } 5^{\mathrm{b}}\end{array}$} & \multirow[t]{2}{*}{$\begin{array}{l}\text { Média } \\
\text { (postos) }\end{array}$} & \multirow{2}{*}{$\begin{array}{c}\text { Teste } \\
\text { Friedman } \\
\text { (p valor) }\end{array}$} \\
\hline & $\mathrm{n}$ & $\%$ & $\mathrm{n}$ & $\%$ & & \\
\hline \multicolumn{7}{|l|}{ A: Assistência na vida cotidiana } \\
\hline Higiene pessoal & 4 & 28,57 & 9 & 64,28 & 4,53 & \\
\hline Medicação & 2 & 14,28 & 11 & 78,57 & 5,28 & \\
\hline Tarefas de casa & 2 & 14,28 & 11 & 78,57 & 5,46 & \\
\hline Compras & 2 & 14,28 & 6 & 42,85 & 3,92 & \\
\hline Preparo o alimento & 2 & 14,28 & 12 & 85,71 & 5,89 & \\
\hline Transporte & 2 & 14,28 & 11 & 78,57 & 5,67 & \\
\hline Cuidados com o dinheiro & 7 & 50,00 & 5 & 35,71 & 3,50 & \\
\hline Ocupação do tempo & 3 & 21,42 & 9 & 64,28 & 5,00 & \\
\hline Consultas médicas & 2 & 14,28 & 11 & 78,57 & 5,71 & \\
\hline \multicolumn{7}{|l|}{ B: Supervisão aos comportamentos problemáticos } \\
\hline Comportamento adverso & 6 & 42,85 & 6 & 42,85 & 5,14 & 9,70 \\
\hline Exigir atenção & 7 & 50,00 & 6 & 42,85 & 4,82 & $(\mathrm{p}>0,05)$ \\
\hline Incomodar as pessoas à noite & 5 & 35,71 & 7 & 50,00 & 5,25 & \\
\hline Comportamento agressivo & 8 & 57,14 & 4 & 28,57 & 4,89 & \\
\hline Comportamento suicida & 8 & 57,14 & 3 & 21,42 & 4,42 & \\
\hline Etilismo & 12 & 85,71 & 2 & 14,28 & 3,46 & \\
\hline Fumar ou beber (bebidas não alcoólicas) & 7 & 50,00 & 3 & 21,42 & 4,82 & \\
\hline Uso de Drogas & 13 & 92,85 & 1 & 7,14 & 3,17 & \\
\hline \multicolumn{7}{|l|}{ D: Impacto nas Rotinas Diárias } \\
\hline Atraso ou cancelamento do compromisso & 5 & 35,71 & 6 & 42,85 & 2,21 & $\begin{array}{c}1,22 \\
(p>0,05)\end{array}$ \\
\hline Alterações nas atividades sociais e de lazer & 5 & 35,71 & 7 & 50,00 & 2,50 & \\
\hline Alterações no serviço e rotina & 3 & 21,42 & 8 & 57,14 & 2,75 & \\
\hline Impedimento à dedicação de outros membros da família & 5 & 35,71 & 7 & 50,00 & 2,53 & \\
\hline
\end{tabular}

Legenda: $\quad{ }^{\text {a }} 1=$ nenhum vez e $2=$ menos que uma vez por semana; ${ }^{\mathrm{b}} 4=$ três a seis vezes por semana e $5=$ todos os dias.

Já a sobrecarga subjetiva causada pela assistência ao cotidiano dos esquizofrênicos foi observada em $28,58 \%$ dos cuidadores que realizavam a higiene pessoal dos pacientes, em $42,86 \%$ dos que administravam os medicamentos, em metade dos cuidadores que precisaram lembrar ou que tiveram de realizar pessoalmente tarefas de casa. Fazer compras $(42,85 \%)$, preparar o alimento $(85,71 \%)$, transportar os doentes $(78,57 \%)$, cuidar do dinheiro recebido pelo doente $(35,71 \%)$, dedicar tempo ao paciente $(64,28 \%)$ e as consultas médicas $(78,57 \%)$ também traziam elevada sobrecarga subjetiva aos cuidadores (Tabela 4).

A sobrecarga objetiva em relação à supervisão dos comportamentos problemáticos foi maior para os aspectos relativos aos comportamentos adversos $(42,85 \%)$, exigir atenção $(42,85 \%)$ e incomodar as pessoas à noite $(50 \%)$ (Tabela 3$)$. Do mesmo modo, quando analisadas tais variáveis em relação à sobrecarga subjetiva (Tabela 4), os aspectos mais importantes foram os comportamentos adversos 
$(57,15 \%)$, exigir atenção $(50 \%)$ e incomodar as pessoas à noite $(64,29 \%)$.

As atividades cotidianas trouxeram sobrecarga objetiva para $42,85 \%$ dos cuidadores, os quais se atrasaram ou cancelaram compromissos em decorrência da doença de seus familiares, para metade daqueles que tiveram alterações nas atividades sociais e de lazer ou sofreram impedimento à dedicação de outros membros da família e para $57,14 \%$ dos cuidadores que sofreram alterações em seu serviço e rotina (Tabela 3). Essas mudanças trouxeram também sobrecarga subjetiva para $85,72 \%$ dessas pessoas (Tabela 4).

Tabela 4. Sobrecarga subjetiva dos familiares cuidadores de portadores de esquizofrenia atendidos em um hospital psiquiátrico de Teresina-PI

\begin{tabular}{|c|c|c|c|c|c|c|c|}
\hline \multirow[t]{2}{*}{ Subescalas } & \multirow[t]{2}{*}{ Variáveis } & \multicolumn{2}{|c|}{$\begin{array}{l}\text { Respostas } \\
1 \text { e } 2^{\mathrm{a}}\end{array}$} & \multicolumn{2}{|c|}{$\begin{array}{c}\text { Respostas } \\
3 \text { e } 4^{\text {b }}\end{array}$} & \multirow{2}{*}{$\begin{array}{c}\text { Média } \\
\text { (postos) }\end{array}$} & \multirow{2}{*}{$\begin{array}{c}\text { Teste } \\
\text { Friedman } \\
\text { (p valor) }\end{array}$} \\
\hline & & n & $\%$ & $\mathbf{n}$ & $\%$ & & \\
\hline \multirow[t]{9}{*}{ A: Assistência na vida cotidiana } & Higiene pessoal & 10 & 71,42 & 4 & 28,58 & 4,64 & \\
\hline & Medicação & 8 & 57,14 & 6 & 42,86 & 4,96 & 6,26 \\
\hline & Tarefas de casa & 7 & 50,00 & 7 & 50,00 & 5,50 & $\mathrm{p}>0,05$ \\
\hline & Compras & 9 & 64,28 & 5 & 35,72 & 5,00 & \\
\hline & Preparo do alimento & 11 & 78,57 & 3 & 21,43 & 4,25 & \\
\hline & Transporte & 6 & 42,85 & 8 & 57,15 & 5,82 & \\
\hline & Cuidados com o dinheiro & 10 & 71,42 & 4 & 28,58 & 4,03 & \\
\hline & Ocupação do tempo & 8 & 57,14 & 5 & 42,86 & 4,89 & \\
\hline & Consultas médicas & 6 & 42,85 & 8 & 57,15 & 5,89 & \\
\hline \multirow[t]{9}{*}{ B: Supervisão aos comportamentos problemáticos } & Comportamento adverso & 6 & 42,85 & 8 & 57,15 & 5,14 & 9,32 \\
\hline & & & & & & & \\
\hline & Exigir atenção & 7 & 50,00 & 7 & 50,00 & 4,75 & \\
\hline & Incomodar as pessoas à noite & 5 & 35,71 & 9 & 64,29 & 5,42 & \\
\hline & Comportamento agressivo & 9 & 64,28 & 5 & 35,72 & 4,42 & \\
\hline & Comportamento suicida & 8 & 57,14 & 6 & 42,86 & 4,75 & \\
\hline & Etilismo & 12 & 85,71 & 2 & 14,29 & 3,60 & \\
\hline & Fumar ou beber (bebidas não alcoólicas) & 8 & 57,14 & 6 & 42,86 & 4,71 & \\
\hline & Uso de Drogas & 13 & 92,85 & 1 & 7,15 & 4,17 & \\
\hline D: Impacto nas Rotinas Diárias & Mudanças na vida do cuidador & 2 & 14,28 & 12 & 85,72 & & \\
\hline
\end{tabular}

Legenda: ${ }^{\mathrm{a}} 1=$ nem um pouco ou nunca e $2=$ muito pouco; ${ }^{\mathrm{b}} 3=$ um pouco e $4=$ muito.

A Tabela 5 apresenta as preocupações dos participantes eram com o futuro $(5,17)$, a segurança cuidadores com os portadores de esquizofrenia. física $(5,07)$ e a saúde $(4,92)$ do paciente $(\mathrm{p}<0,05)$.

Observa-se que as maiores preocupações dos

Tabela 5. Sobrecarga Subjetiva Referente à Preocupação com o Paciente em Familiares Cuidadores de Portadores de Esquizofrenia Atendidos em um Hospital Psiquiátrico de Teresina-PI

\begin{tabular}{|c|c|c|c|c|c|c|c|}
\hline \multirow[t]{2}{*}{ Subescalas } & \multirow[t]{2}{*}{ Variáveis } & \multicolumn{2}{|c|}{$\begin{array}{c}\text { Respostas } \\
1 \text { e } 2^{\mathrm{a}} \\
\end{array}$} & \multicolumn{2}{|c|}{$\begin{array}{c}\text { Respostas } \\
4 \text { e } 5^{b} \\
\end{array}$} & \multirow{2}{*}{$\begin{array}{c}\text { Média } \\
\text { (postos) }\end{array}$} & \multirow{2}{*}{$\begin{array}{c}\text { Teste } \\
\text { Friedman } \\
\text { (p valor) }\end{array}$} \\
\hline & & $\mathrm{n}$ & $\%$ & $\mathrm{n}$ & $\%$ & & \\
\hline \multirow[t]{8}{*}{ E: Preocupações com os pacientes } & Segurança física & 0 & 0 & 14 & 100,00 & 5,07 & \\
\hline & Qualidade do tratamento & 7 & 50,00 & 5 & 35,71 & 2,14 & 26,34 \\
\hline & & & & & & & $\mathrm{p}<0,001$ \\
\hline & Vida social & 1 & 7,14 & 6 & 42,86 & 3,35 & \\
\hline & Saúde & 0 & 0 & 14 & 100,00 & 4,92 & \\
\hline & Moradia & 5 & 35,71 & 6 & 42,86 & 2,85 & \\
\hline & Finanças & 2 & 14,28 & 12 & 85,71 & 4,46 & \\
\hline & Futuro & 0 & 0 & 14 & 100,00 & 5,17 & \\
\hline
\end{tabular}

Legenda: ${ }^{\mathrm{a}} 1=$ nunca e $2=$ raramente; ${ }^{\mathrm{b}} 4=$ frequentemente e $5=$ sempre ou quase sempre. 


\section{DISCUSSÃO}

A esquizofrenia tem o status de uma síndrome clínica e pode incluir uma série de patologias específicas, e, alternativamente, pode ser uma única doença com manifestações bastante variáveis entre os casos (Carpenter, 2007).

Os fatores biológicos relacionados à síndrome são representados por fatores de risco genéticos, anormalidades morfoanatômicas (eventualmente causadas por lesão estrutural) e mau funcionamento de sistemas neurotransmissores. Os principais fatores psicossociais são relacionados às manifestações psicogênicas e à interação do sujeito afetado com o ambiente social, envolvendo dimensões como ansiedade muito intensa, estado de estresse elevado, fobia social e situações sociais e emocionais intensas (Paixão et al., 2009).

Os resultados deste estudo demonstram que, em sua maior parte, os cuidadores de adultos portadores de esquizofrenia avaliados são do sexo feminino $(71,42 \%)$ e em sua maioria $(85,71 \%)$, casados (tabela 1). Estudos apontam que as relações de cuidado foram historicamente construídas por meio da divisão de gênero no trabalho, em que se buscou determinar lugares sociais de acordo com o gênero de cada sujeito. Essa divisão por sexo instituiu-se socialmente pela fusão que se estabeleceu entre o social e o biológico por intermédio do corpo feminino, construindo-se assim "o mito do amor materno", pelo qual o cuidar tornou-se uma função naturalmente feminina (Banditer, 1985).

A análise dos dados revelou que a maioria dos pacientes tinha diagnóstico estabelecido havia algum tempo - em média, 11,54 anos $( \pm 0,08)$, com o maior tempo de diagnóstico de esquizofrenia de 26 anos, o que pode justificar a grande sobrecarga incidente sobre os cuidadores. Tal achado está em concordância com os do estudo de Cardoso et al. (2006), em que os autores concluem que quanto maior a duração da esquizofrenia, maior o comprometimento na qualidade de vida do paciente.

Além disso, metade dos familiares cuidadores cursara apenas o ensino fundamental completo, evidenciando um baixo nível de escolarização dos cuidadores, os quais por isso podem ter relativa dificuldade em entender corretamente a esquizofrenia. Adicionalmente, considerando-se que mais da metade dos entrevistados provinha do Maranhão e do Pará, destaca-se a necessidade de acolhimento, por parte dos profissionais e das instituições psiquiátricas, àquelas pessoas que se encontram física e existencialmente fragilizadas, como também de fornecer apoio aos familiares. Este acolhimento fomenta a adesão do sujeito ao tratamento proposto, ajudando-o a se sentir cuidado, protegido e visto como um indivíduo que merece respeito e atenção (Stockinger, 2007).

A grande dependência dos pacientes para realizar atividades de vida diária como pentear o cabelo, tomar banho e vestir-se encontrada neste estudo representa um indicativo real de sobrecarga objetiva da maioria desses familiares. Ao analisar as respostas dos cuidadores ao lhes ser perguntado qual o incômodo que essas atividades representavam, foi constatado que quase um terço dos cuidadores apresentava sobrecarga subjetiva elevada. Os portadores de esquizofrenia tendem a apresentar perturbações no comportamento funcional referentes aos cuidados pessoais, apresentando fracos hábitos de higiene e arrumação pessoal (Kaplan, 1997), e durante o período de crise o paciente necessita de cuidados higiênicos frequentes, devido à perda de algumas habilidades psicomotoras que fazem o paciente exigir cotidianamente cuidados físicos e higiênicos (Rosa, 2008).

Outro aspecto que representou elevada sobrecarga objetiva e subjetiva para os familiares avaliados foi a necessidade de lembrar ou encorajar o paciente a tomar seus medicamentos, dar o medicamento pessoalmente ou às escondidas. É notório que a falta de adesão ao tratamento medicamentoso entre portadores de esquizofrenia é elevada e deve ser considerada em qualquer programa de tratamento (Rosa, Marcolin \& Elkis, 2005). Também deve ser destacado que as dificuldades no cuidado desses indivíduos resultam não apenas dos efeitos indesejáveis da farmacoterapia, mas também do fato de a pessoa com comprometimento mental tender a não se aceitar como doente e passar a não assumir o tratamento medicamentoso (Rosa, 2008).

Considerando-se que a esquizofrenia é uma síndrome crônica complexa e que aos familiares cabe cuidar do membro da família doente, que pode ficar dependente e desorganizado, parece difícil dizer o que representa para a família ter um de seus membros portador dessa doença (Teixeira, 2005).

Embora 85,71\% dos cuidadores apresentassem elevada sobrecarga objetiva ao cozinhar ou preparar as refeições todos os dias, ou ainda, dar o alimento diretamente ao paciente quando este se encontrava impossibilitado devido aos sintomas depressivos da esquizofrenia ou acamado, tais aspectos representavam sobrecarga subjetiva para pouco mais de $20 \%$ dos cuidadores, pois os familiares preparavam o alimento não apenas para os pacientes esquizofrênicos, mas também para os outros membros da família. 
Mais da metade dos cuidadores referiram sentir-se incomodados em ter que levar o seu parente a algum lugar, por não confiarem em outra pessoa para cuidar do paciente, ou ainda em decorrência do apego do paciente ao seu cuidador, tendo este último que assumir a responsabilidade total pelo auxílio prestado ao parente esquizofrênico. Neste sentido, estudos têm demonstrado que alterações de comportamento sofridas pelos pacientes esquizofrênicos como impulsividade, agitação ou lentidão psicomotora, comportamento bizarro, estupor, falta de destreza, rigidez nos movimentos corporais e sintomas depressivos, associados com a ociosidade, têm um papel decisivo na ativação da hipervigilância de alguns familiares (Souza, Guimarães \& Ballone, 2004; Rosa, 2008).

Tratando-se dos cuidados referentes ao dinheiro do paciente, a maioria dos cuidadores relatou que, como o dinheiro estava em seu poder, isso não lhes causava nenhuma preocupação ou sacrifício.

Assim, no presente estudo o fato de ajudar, lembrar ou encorajar o paciente a se ocupar com alguma coisa, sugerir que leia uma revista ou visite pessoas, usar o tempo para trabalhar ou estudar representou sobrecarga objetiva para $64,28 \%$ dos cuidadores. Insistir em que os doentes não fiquem ociosos ou com pensamentos negativos trouxe sentimento de sobrecarga subjetiva a $42,86 \%$ dos entrevistados. É importante destacar que os indivíduos esquizofrênicos têm recursos pessoais limitados quanto às habilidades sociais e cognitivas restritas e habilidades ambientais, como, por exemplo, pobreza e ausência de empregos adequados (Souza \& Coutinho, 2006).

No que se refere à ajuda prestada pelos cuidadores em ter que levar o portador de esquizofrenia às consultas médicas ou às atividades nos serviços de saúde mental, tais atividades representaram sobrecarga objetiva para $78,57 \%$ dos participantes que prestam esse auxílio numa frequência acima de três vezes por semana ou todos os dias, atividade com a qual mais da metade dos cuidadores referiram ficar incomodados. $\mathrm{Na}$ instituição em que foi realizada a pesquisa, o familiar deve acompanhar o paciente diariamente ao hospital para que este seja medicado e/ou consultado, o que explica a elevada frequência nessa variável.

Os comportamentos problemáticos dos pacientes não representavam incômodo devido à baixa frequência com que ocorriam, segundo os cuidadores. De fato, Villares (2000) cita que a família tem um lugar e uma função centrais na vida dos portadores de esquizofrenia, os quais, nessa convivência, aprendem a enfrentar momentos de agravamento do quadro sintomatológico e a manejar situações de inatividade, depressão, agressividade, confusão, desorganização e inadequação. Além disso, o cuidador aprende, com a observação e vivência guiada pelo ensaio e erro, a aplacar, mesmo que de forma mínima, a manifestação da enfermidade (Rosa, 2008).

Metade dos familiares referiu precisar evitar ou impedir que o paciente incomodasse as pessoas durante a noite numa frequência acima de três vezes ou diária, e para 64,29\%, lidar com essas ações do paciente representava elevada sobrecarga subjetiva. Desse modo, destaca-se que a exacerbação psicomotora, a agitação e a insônia do paciente em crise aguda fizeram desenvolver-se essa inquietude no comportamento, trazendo com isso a insatisfação do familiar.

Alucinações visuais e auditivas estão entre os sintomas mais observados na crise esquizofrênica. Além disso, os pacientes podem apresentar pensamento desorganizado e comportamento catatônico como sintomas adicionais, e o diagnóstico da esquizofrenai é feito com base principalmente nos distúrbios de expressão e de comportamento (Burgy, 2008).

A maior parte dos sujeitos da pesquisa referiu elevada sobrecarga objetiva em suas rotinas diárias. Quando um dos membros da família adoece, os cuidadores sofrem alterações nas suas atividades sociais normais e suas tarefas precisam ser desenvolvidas por outras pessoas, pois o familiar necessita dedicar-se ao cuidado do doente (Teixeira, 2005). Foi constatado que todos os familiares confirmaram mudanças quase permanentes na sua rotina diária, no seu trabalho ou na sua vida social, e a maioria relatou elevada sobrecarga subjetiva.

Grande parte desses resultados corrobora o estudo realizado por Barroso, Bandeira e Nascimento (2007), em que os autores apontam a necessidade constante de o cuidador realizar tais tarefas. Além disso, com o advento da reforma psiquiátrica, passaram a fazer parte do cotidiano dos familiares atividades como atender às necessidades básicas dos pacientes, coordenar suas atividades diárias, administrar sua medicação, acompanhá-los aos serviços de saúde, lidar com seus comportamentos problemáticos e episódios de crise, fornecer-lhes suporte social, arcar com gastos e superar as dificuldades dessas tarefas (Teixeira, 2005).

No item referente à preocupação com o paciente, os participantes apresentaram maior preocupação com o futuro do paciente $(5,17)(\mathrm{p}<0,05)$. Os familiares temiam que algo ruim acontecesse aos doentes, que 
sofressem acidente, entrassem em uma briga ou que alguém se aproveitasse da doença. Estudos mostram que os familiares cuidadores de pacientes esquizofrênicos se preocupavam frequentemente com o futuro dos pacientes, em especial sobre como seriam suas vidas após a morte dos cuidadores atuais (Barroso et al., 2007).

Shirakawa (2000) afirma que toda doença crônica dificulta a vida do portador e sua relação com a família, acrescentando que a loucura afeta não só o indivíduo doente, mas toda a família e ainda a rede de relações sociais. Os sintomas produzem distorções nas relações humanas, de modo que, após lidar com a psicose por vários anos, tanto o indivíduo como as pessoas mais próximas se modificam radicalmente.

A responsabilidade por gerir cuidados a adultos portadores de esquizofrenia deflagrou um sentimento de sobrecarga nas pessoas que assumiram esse papel de cuidador no presente estudo. As famílias estudadas apresentam uma série de dificuldades físicas, sociais e emocionais diante dessa síndrome, especialmente pelo fato de que suas necessidades de apoio, orientação e preparação para o enfrentamento da esquizofrenia ainda não estão sendo atendidas pela instituição psiquiátrica pesquisada. Esses resultados são concordantes com os estudos de Barroso et al. (2007), segundo os quais prover cuidados a paciente psiquiátricos gera o sentimento de sobrecarga, pois o cuidador sofre mudanças em sua rotina de vida, deixando de satisfazer suas necessidades para atender as necessidades do paciente.

Desse modo, faz-se necessário repensar os serviços prestados pelas instituições em saúde mental, de forma a ajudar os familiares a lidar com esta complexa enfermidade por meio da implantação de grupos psicoeducativos, de visitas domiciliares, de atividades ocupacionais e de oficinas de artes, de forma a fornecer mais informações sobre a esquizofrenia e melhorar a relação entre familiar e paciente. Enfim, é preciso propor ações concretas que possam, em médio e longo prazo, reduzir significativamente o surgimento do sentimento de sobrecarga vivenciado pelos familiares de adultos portadores de esquizofrenia.

\section{CONCLUSÃO}

Os resultados deste estudo podem contribuir de forma indireta para a ampliação dos saberes a respeito da sobrecarga em familiares cuidadores de portadores de distúrbios mentais, instigando uma maior reflexão sobre as condições dos programas e das políticas em saúde mental em relação à criação de medidas voltadas para o acompanhamento dos cuidadores de pacientes esquizofrênicos. $\mathrm{O}$ cuidado intenso e contínuo torna-se um fardo para os cuidadores de portadores de esquizofrenia, evidenciando a necessidade de um trabalho contextualizado com a realidade das famílias, em que seja oferecido um espaço para os cuidadores externarem seus conflitos emocionais, situações de crise e dúvidas, para que desta forma eles possam ter suporte psicológico para enfrentar as adversidades que surgem no cuidado cotidiano com o paciente.

Desse modo, destaca-se a necessidade de buscar novas estratégias de enfrentamento na atenção psiquiátrica, voltadas não somente para a atenção ao paciente, mas também ao cuidador. Tais estratégias devem incluir intervenções psicossociais no sentindo de promover acolhimento, empatia e escuta terapêutica para levar o cuidador a entrar em contato com seus sentimentos, de forma a experienciar e descobrir novas possibilidades de existir e se reconhecer como um ser multifacetado. Acredita-se que essas intervenções podem contribuir para reduzir as sobrecargas objetivas e subjetivas nas dimensões relacionadas à assistência na vida cotidiana, à supervisão de comportamentos problemáticos, ao impacto nas rotinas diárias e preocupações com o paciente, diminuindo o efeito desse cuidado em seu viver e, consequentemente, melhorando sua qualidade de vida.

\section{REFERÊNCIAS}

Ayres, M., Ayres Jr, M., Ayres, D. L. \& Santos, A. A. S. (2007). Bioestat 5.0. Sociedade Civil Mamirauá.

Badinter, E. (1985). Um amor conquistado: $O$ mito do amor materno. Rio de Janeiro, RJ: Nova Fronteira.

Bandeira, M. \& Barroso, S. (2005). Sobrecarga das famílias de pacientes psiquiátricos. Jornal Brasileiro de Psiquiatria, 54(1), 34-46.

Bandeira, M., Calzavara, M. G. P. \& Varella, A. A. B. (2005). Escala de Sobrecarga dos familiares de pacientes psiquiátricosFBIS-BR: Adaptação transcultural para o Brasil. Jornal Brasileiro de Psiquiatria, 54(3), 206-214.

Barroso, S. M., Bandeira, M. \& Nascimento, E. (2007). Sobrecarga de familiares de pacientes psiquiátricos atendidos na rede pública. Revista de Psiquiatria Clínica, 34(6), 270-277.

Bentall, R. P. (2006). Schizophrenia: Challenging the Orthodox. The British Journal of Psychiatry, 188(3), 296-297.

Brasil, Ministério da Saúde (1996). Resolução 196/96 do Conselho Nacional de Saúde/MS sobre Diretrizes e Normas Regulamentadoras de Pesquisa envolvendo seres humanos. Diário Oficial da União.

Burgy, M. (2008). The Concept of Psychosis: Historical and Phenomenological Aspects. Schizophr Bull, 34(6), 1200-1210. 
Cardoso, C. S., Caiaffa, W. T., Bandeira, M., Siqueira, A. L., Abreu, M. N. S. \& Fonseca, J. O. P. (2006). Qualidade de vida e dimensão ocupacional na esquizofrenia: uma comparação por sexo. Caderna de Saúde Pública, 22(6), 1303-1314.

Carpenter, W. T. (2007). Schizophrenia: Disease, Syndrome, or Dimensions? Family Process, 46, 199-206.

Crow, T. J. (1980). Molecular pathology of schizophrenia: more than one disease process? British Medical Journal, 280(6207), 66-68.

Dean, A. G., Dean, J. A., Burton, A. H. \& Dicher, R. C. (1994). Epi Info, Version 6: A Word Processing, Database, and Statistics Program for Epidemiology on Micro-Computers. Atlanta: Centers for Disease Control and Prevention.

Kaplan, H. I. (1997). Compêndio de psiquiatria: ciência do comportamento e psiquiatria clínica, 7 ed. Porto Alegre: Artes Médicas.

Paixão, C., Matias, D., Alencar, I., Nunes, M., Sales, P. \& Veiga, P. H. A. (2009) Análise da prevalência dos transtornos psíquicos na região metropolitana do Recife. Ciência \& Saúde Coletiva, 14(1), 261-266.

Pegoraro, R. F. \& Caldana, R. H. L. (2006). Sobrecarga de Familiares de Usuários de um Centro de Atenção Psicossocial. Psicologia em estudo, 11(3), 569-577.

Rosa, L. C. S. (2008). Transtorno Mental e o cuidado na Família. São Paulo: Cortez.

Rosa, M. A., Marcolin, M. A. \& Elkis, H. (2005). Evaluation of the factors interfering with drug treatment compliance among Brazilian patients with schizophrenia. Revista Brasileira de Psiquiatria, 27(3), 178-184.
Scazufca, M. (2000). Abordagem familiar em esquizofrenia. Revista Brasileira de Psiquiatria, 22,50-52.

Schene, A. H., Tessler, R. C. \& Ganache, G. M. (1994). Instruments measuring family or caregiver burden in severe mental illness. Social Psychiatry and Psychiatric Epidemiology, 29, 228-240.

Shirakawa, I. (2000). Aspectos gerais do manejo do tratamento de pacientes com esquizofrenia. Revista Brasileira de Psiquiatria, $22,56-58$.

Souza, J. C., Guimarães, L. A. M. \& Ballone, G. J. (2004). Psicopatologia e psiquiatria básicas. São Paulo: Vetor: UCDB.

Souza, L. A. \& Coutinho, E. S. F (2006). Fatores associados à qualidade de vida de pacientes com esquizofrenia. Revista Brasileira de Psiquiatria, 28(1), 50-58.

Stockinger, R. C. (2007). Reforma Psiquiátrica Brasileira: Perspectivas humanistas e existenciais. Petrópolis, RJ: Vozes.

Teixeira, M. B. (2005). Qualidade de vida de familiares cuidadores do doente esquizofrênico. Revista Brasileira de Enfermagem, 58(2), 171-175.

Villares, C. C. (2000). Adaptação transcultural de intervenções psicossociais na esquizofrenia. Revista Brasileira de Psiquiatria. 22, 53-55.
Endereço para correspondência:
Manoel Dias de Souza Filho. Rua Equador, $n^{\circ}$ 118, Conjunto Jardim Esperança 3, Bairro Ceará, CEP 64215-620, Parnaíba-PI, Brasil. E-mail: carminhacmartins@yahoo.com.br. 